\title{
Bilateral pneumothorax, pneumonia, and pneumomediastinum after injection of a hard drug into the neck
}

\author{
Pier Luigi Filosso, MD, Anna Arslanian, MD, Enrico Ruffini, MD, and Maurizio Mancuso, MD, Torino, Italy
}

A

ir is facilitated to enter the neck with injection, and it can produce subcutaneous emphysema. Sometimes pneumomediastinum and pneumothorax can occur if air penetrates into the mediastinal and pleural spaces, respectively. If injection is performed at the base of the neck or if the patient experiences a glottis edema after this procedure, pneumothorax is a common complication. In patients with immunodeficiency, injection of a hard drug into the neck can occasionally be complicated by local or extended infection, including descending necrotizing mediastinitis.

We report a case of subcutaneous emphysema, pneumomediastinum, bilateral pneumothorax, and pneumonia in a 34 -year-old white woman after a single injection of a hard drug into the neck.

\section{Clinical Summary}

A 34-year-old white woman who was addicted to hard drugs was referred to us for acute dyspnea 18 hours after injection of a hard drug into her neck. Apparently the patient was in good general condition before the injection. The patient had a right laterocervical swelling (Figure 1) and subcutaneous cervical and thoracic emphysema.

A chest $\mathrm{x}$-ray film showed bilateral pneumothorax (more evident at the left side), pneumomediastinum, and bilateral parenchymal condensations (more evident at the right side), which were suggestive of bilateral pneumonia; this was confirmed by means of thoracic computed tomography (Figures 2, 3, and 4).

A bronchoscopic examination showed a dense bilateral purulent secretion, which was aspirated, without any tracheal or bronchial lesion. Staphylococcus aureus and Candida albicans were cultured.

Bilateral pneumothorax was treated with 2 chest tubes. Surgical exploration of the neck through a U-shaped cervicotomy was performed, and no pus or blood were observed. The mediastinal space was drained, with 2 drainages in the paratracheal space. Air, but not purulent fluid, was collected.

The postoperative course was complicated by respiratory insufficiency caused by bilateral pneumonia, which necessitated

\footnotetext{
From the Department of Thoracic Surgery, University of Torino, and San Giovanni Battista Hospital, Torino, Italy.

Received for publication Feb 6, 2002; accepted for publication March 6, 2002.

Address for reprints: Pier Luigi Filosso, MD, University of Torino (Italy), Department of Thoracic Surgery, San Giovanni Battista Hospital, Via Genova, 3, 10126 Torino, Italy (E-mail: pierluigifilosso@tiscalinet.it).

J Thorac Cardiovasc Surg 2002;124:1233-4

Copyright (C) 2002 by The American Association for Thoracic Surgery

$0022-5223 / 2002 \$ 35.00+0 \quad \mathbf{1 2 / 5 4 / 1 2 4 4 9 4}$

doi:10.1067/mtc.2002.124494
}

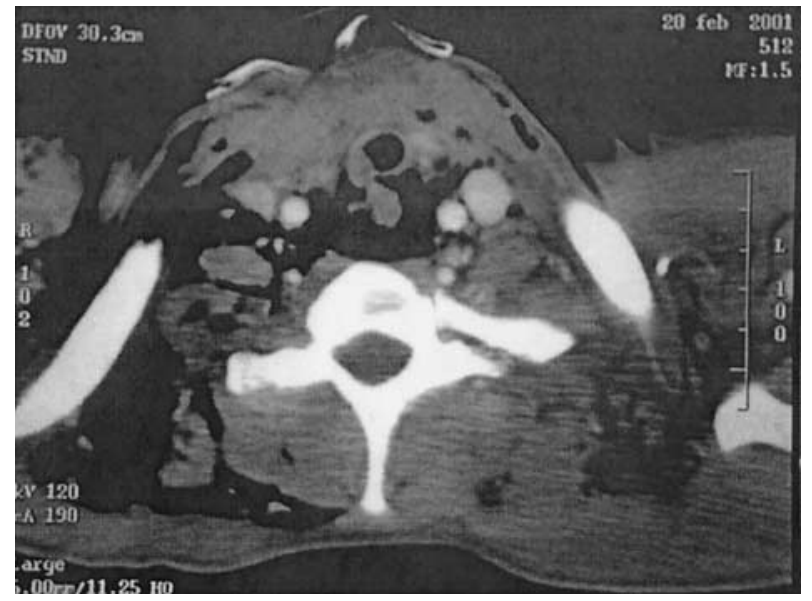

Figure 1. Neck computed tomographic scan showing a right laterocervical and paratracheal swelling.

intensive care treatment for 8 days. Pneumothorax and pneumomediastinum resolved in 6 days, and the patient was discharged from the hospital on the 15 th postoperative day.

\section{Discussion}

Although rare, pneumomediastinum might result from entry of air into the mediastinal space through the neck, trachea, bronchi, esophagus, retroperitoneum, and abdomen. ${ }^{1}$ Diagnosis and management of pneumomediastinum is an important topic for a thoracic surgeon because of the potentially life-threatening consequences.

The neck can be anatomically divided into parapharyngeal, prevertebral, and retropharyngeal spaces; these spaces contain loose fatty connective tissue enclosed by facial planes. ${ }^{2}$ When trauma occurs, these tissues break down, and their place is taken by air or pus (eg, in case of necrotizing fasciitis). ${ }^{3}$ Through mediastinum, air can reach the pleural space, resulting in pneumothorax

In this case we hypothesized that pneumomediastinum and bilateral pneumothorax were due to an exceptional increase of endothoracic pressure. Many reports in the literature describe pneumomediastinum caused by cough or asthmatic crisis ${ }^{4-6}$; we hypothesized that the drug injection in the neck was responsible for an acute transient respiratory obstruction that produced pneumomediastinum and pneumothorax. The patient had no clinical evidence of pneumonia or pulmonary diseases before the referral, which might theoretically have been responsible for pneumomediastinum and pneumothorax.

If respiratory insufficiency develops, mediastinal space drainage is to be performed. In case of pneumomediastinum with pneumothorax, surgical treatment is mandatory. 


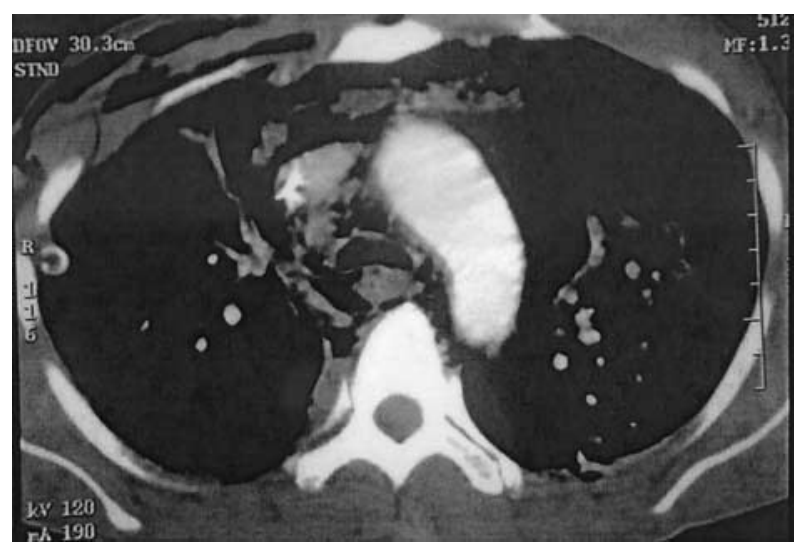

Figure 2. Thoracic computed tomographic scan showing large pneumomediastinum.

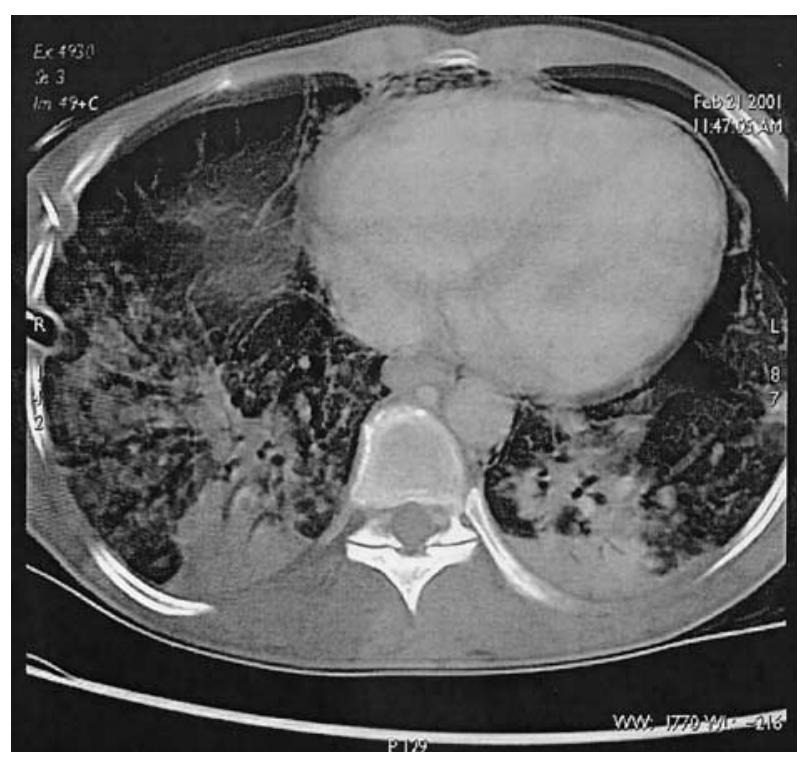

Figure 3. Thoracic computed tomographic scan with bilateral pneumothorax and pneumonia.

In this patient we first drained the pleural space, and then we performed a cervical exploration to discover and treat possible purulent foci in the neck (the site of drug's injection) because of the high risk of descending mediastinitis. The presence of signif-

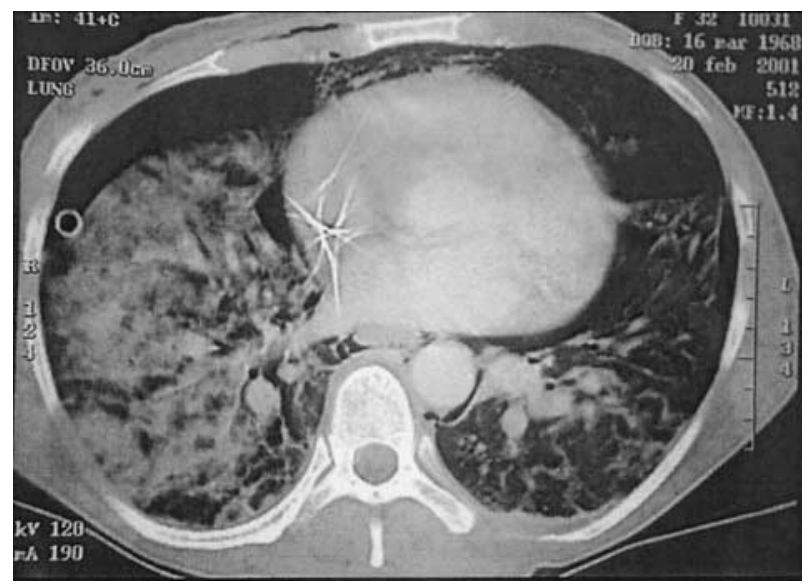

Figure 4. Thoracic computed tomographic scan with massive right pneumonia.

icant associated comorbidity factors and the absence of pus in the mediastinum induced us to adopt a strategy of simple drainage of the mediastinum through 2 paratracheal tubes instead of major mediastinal surgical exploration.

In drug addicts bilateral pneumonia is frequently observed, and an hematogenous cause is frequent. Staphylococcus aureus, Staphylococcus epidermidis, Enterobacteriaceae, and Candida albicans are commonly observed, as in the case we describe.

We thank Domenica Garabello, MD, and Ottavio Davini, MD, (Radiological Service, San Giovanni Battista Hospital) for the radiologic images and for their consultations.

\section{References}

1. Hoover LR, Febinger DL, Tripp HF. Rhinolalia: an underappreciated sign of pneumomediastinum. Ann Thorac Surg. 2000;69:615-6.

2. Eliachar I, Peleg H, Joachims HZ. Mediastinitis and bilateral pyopneumothorax complicating a parapharyngeal abscess. Head Neck Surg. 1981;3:438-42.

3. Levitt GW. Cervical fascia and deep neck infections. Laryngoscope. 1970;80:409-35.

4. Abolnik I, Lossos IS, Breuer R. Spontaneous pneumomediastinum. A report of 25 cases. Chest. 1991;100:93-5.

5. Yellin A, Gapany-Gapanavicius M, Lieberman Y. Spontaneous pneumomediastinum: is it a rare cause of chest pain? Thorax. 1983;38: 383-5.

6. Rose WD, Veach JS, Tehranzdeh J. Spontaneous pneumomediastinum as a cause of neck pain, dysphagia and chest pain. Arch Intern Med. 1984;144:392-3. 